\title{
Ecoinovações: um ensaio teórico sobre conceituação, determinantes e achados na literatura
}

Eco-innovation: a theoretical on concept, determining and found in the literature

\author{
Eco-innovation: une partie théorique sur le concept, détermination et trouve \\ dans la littérature
}

Eco-innovación: un concepto en teórico, determinación y encontrados en la literatura

\author{
Pedro Gilberto Aloise* \\ (pedroaloise@hotmail.com) \\ Cristine Hermann Nodari** \\ (cristine.nodari@gmail.com) \\ Eric Charles Henri Dorion* \\ (echdorion@gmail.com)
}

Recebido em 14/07/2015; revisado e aprovado em 17/09/2015; aceito em 07/12/2015 DOI: http://dx.doi.org/10.20435/1984042X2016211

\begin{abstract}
Resumo: Esta pesquisa teve por objetivo identificar as principais características e os fatores direcionadores das ecoinovações, também, denominadas de inovações verdes, inovações ambientais ou inovações sustentáveis. A literatura que fundamentou este trabalho foi articulada a partir de uma pesquisa documental, com análise bibliométrica de artigos. Dentre os achados, foi possível identificar particularidades relacionadas às inovações ambientais que não comprovam totalmente algumas teorias, amplamente aceitas no meio acadêmico.

Palavras-chave: inovações; eco-inovações; bibliometria.

Abstract: This research aimed to identify the main characteristics and factors drivers of eco-innovations also called green innovations, environmental innovations or sustainable innovations. The literature that justified this work was articulated from a documentary research, with bibliometric analysis articles. Among the findings it was possible to identify characteristics related to environmental innovations that do not fully prove some theories, widely accepted in academia.

Key words: innovations; eco-innovations; bibliometrics.

Résumé: Cette recherche visait à identifier les principales caractéristiques et facteurs pilotes d'éco-innovations aussi appelés innovations vertes, les innovations environnementales ou d'innovations durables. La littérature qui a justifié ce travail a été articulé a partir d'une recherche documentaire, avec des articles d'analyse bibliométriques. Parmi les conclusions était possible d'identifier les caractéristiques liées à des innovations environnementales qui ne prouvent pas complètement certaines théories, largement acceptées dans le milieu universitaire.

Mots-clés: innovations; les éco-innovations; la bibliométrie.

Resumen: Esta investigación tuvo como objetivo identificar las principales características y factores conductores de innovaciones ecológicas también llamadas innovaciones verdes, innovaciones ambientales o innovaciones sostenibles. La literatura que justifica este trabajo se articula a partir de una investigación documental, con artículos de análisis bibliométricos. Entre los hallazgos fue posible identificar las características relacionadas con las innovaciones ambientales que no acrediten totalmente algunas teorías, de amplia aceptación en el mundo académico.

Palabras clave: innovaciones; innovaciones ecológicas; bibliometría.
\end{abstract}

\section{INTRODUÇÃO}

A relevância da inovação, como tema de estudo e pesquisa acadêmica, foi reconhecida a partir das ideias do economista austríaco Joseph Alois Schumpeter, que a considerava condição necessária para o desenvolvimento econômico, proporcionando dinamismo às atividades produtivas, uma vez que inovar trazia, implicitamente, a ideia das firmas produzirem novos produtos ou os mesmos produtos de diferentes maneiras e com combinações de recursos e fatores de produção. Ao ser copiado ou imitado pelos concorrentes, o empresário promove outras inovações, materializando novas ideias e servindo-se de novas regras. A busca pela criação contínua, gerando novos ciclos de negócio, foi denominada "destruição criadora" (SCHUMPETER, 1961).

\footnotetext{
* Universidade de Caxias do Sul (UCS), Caxias do Sul, Rio Grande do Sul, Brasil.

** Universidade Potiguar (UnP), Natal, Rio Grande do Norte, Brasil.
} 
A partir das ideias propostas por Schumpeter, a Organização para Cooperação e Desenvolvimento Econômico (OCDE, 2012) elaborou a padronização e sistematização do conceito e demais temas atrelados à inovação, através de publicação na qual formalizou os resultados dos encontros promovidos pela entidade, com vistas a estabelecer diretrizes para coleta e interpretação de dados sobre inovação. O referido documento, denominado Manual de Oslo, em sua terceira edição, definiu inovação como sendo a implementação de um produto (bem ou serviço) novo ou, significativamente, melhorado, ou um processo, ou um novo método de marketing, ou um novo método organizacional nas práticas de negócios, na organização do local de trabalho ou nas relações externas (OCDE, 1997). Nesse mesmo documento, classificaram-se as inovações em quatro tipos, assim definidos: i) inovações de produto que envolvem mudanças significativas nas potencialidades de produtos e serviços. Incluem-se bens e serviços, totalmente, novos e aperfeiçoamentos importantes para produtos existentes; ii) inovações de processo que representam mudanças significativas nos métodos de produção e de distribuição; iii) inovações organizacionais que se referem à implementação de novos métodos organizacionais, tais como mudanças em práticas de negócios, na organização do local de trabalho ou nas relações externas da empresa; iv) inovações de marketing que envolvem a implementação de novos métodos de marketing, incluindo mudanças no design do produto e na embalagem, na promoção do produto e sua colocação e em métodos de estabelecimento de preços de bens e de serviços.

Os estudos sobre inovação, a partir do Relatório Our Common Future produzido pela World Commission on Environment and Developmente coordenado por Gro Brundtland (1987), passaram a ter uma significação mais profunda, não apenas para a atividade econômica e para a gestão dos negócios, mas também para o aproveitamento e uso mais racional e eficiente dos recursos naturais. Para o autor, ao abordar a preocupação com o meio ambiente e o desenvolvimento sustentável do planeta, reconhece-se a importância da inovação no desenvolvimento de novos produtos e processos, com vistas à utilização mais racional dos recursos naturais. Os resul- tados advindos dessa nova abordagem não somente impactaram variáveis de natureza econômica, tecnológica e organizacional, mas também trouxeram uma preocupação com o meio ambiente, conferindo às inovações uma nova dimensão: a responsabilidade social.

Assim, as inovações voltadas para as questões da sustentabilidade passaram a ser denominadas, no meio científico internacional (WAGNER, 2010; REID; MIEDZINSKI, 2008), como ecoinovações ou inovações ecológicas (ecoinnovations), inovações ambientais (environmental innovations), inovações sustentáveis (sustainable innovations) ou inovações verdes (green innovations). Boons e LüdekeFreund (2013) atribuíram o fato de os termos ecoinnovation e sustainable innovation serem intercambiáveis, à sua utilização por pesquisadores de diferentes áreas.

Este trabalho de natureza teórica visou a identificar, na literatura existente, as principais características e os fatores direcionadores e determinantes das ecoinovações que levaram ao desenvolvimento sustentável. Em seu Report of the World Commission on Environment and Development: Our Common Future, Brundtland (1987) afirmou a existência de um desenvolvimento sustentável, no qual a satisfação das necessidades das atuais gerações não comprometesse a capacidade de as gerações futuras satisfazerem as suas necessidades. Nesse sentido, as práticas de inovação incorporam as premissas da sustentabilidade, pois, além do desenvolvimento de novos produtos e serviços, da satisfação das necessidades do cliente, da criação de vantagens competitivas para as empresas e demais medidas de desempenho daí decorrentes, devem levar em consideração questões como o consumo de energia, degradação do meio ambiente, aproveitamento racional dos recursos naturais e matérias-primas, geração de resíduos, poluição, dentre outros, qualificando novas formas de inovação, denominadas inovações ecológicas, sustentáveis, ambientais ou "verdes". Tomando-se Brundland (1987) como um dos referenciais de partida, constata-se que são recentes as questões de pesquisa e os estudos organizacionais relacionados às inovações ambientais, sustentabilidade e desenvolvimento sustentável, dentre os quais podemos exemplificar: i) a relação entre as inovações ambientais como fontes de 
vantagem competitiva e incremento da competitividade (PORTER; VAN DER LINDE, 1995a; 1995b; NIDUMOLU; PRAHALAD; RANGASWAMI, 2009; SEVERO et al., 2015); ii) a adequação dos modelos de negócios das práticas sustentáveis (FADHILAH; RAMAYAH, 2012; OECD, 2012; BOONS et al., 2013; BOONS; LÜDEKE-FREUND, 2013); iii) ecoeficiência nas práticas de negócios, incluindo produção sustentável, análise do ciclo de vida e gestão verde da cadeia de suprimentos (WBCSD, 2000; GMELIN; SEURING, 2014; OECD, 2009b; SEMAN et al., 2012); iv) desenvolvimento de novos produtos sustentáveis (BAUMANN et al., 2002; HUANG; WU, 2010; MEDEIROS et al., 2012; GMELIN; SEURING, 2014).

O artigo foi organizado e dividido, além dessa seção introdutória, em mais quatro seções. A próxima seção discorre sobre a revisão da literatura, envolvendo os temas de pesquisa. A seção seguinte, ou seja, a terceira, apresenta os procedimentos metodológicos adotados na condução da pesquisa. A quarta seção expõe a discussão e a apresentação dos resultados alcançados com o levantamento teórico. A quinta seção, por fim, apresenta as considerações finais e oportunidades de trabalhos futuros.

\section{REFERENCIAL TEÓRICO}

\subsection{Conceito, características e classificação das inovações sustentáveis}

Conforme afirmaram Schiedering et al. (2012), ao realizarem uma revisão da literatura sobre o tema e ao compararem a definição de sustentabilidade ou sustainability de Brundtland (1987) com as demais denominações (eco, environmental e green innovation), concluíram que, ao serem desenvolvidas essas modalidades de inovações, deveriam ser levados em consideração os aspectos econômicos, ecológicos e sociais decorrentes das inovações. Essas dimensões constituem o tripé da sustentabilidade ou triple-botton-line, conceito esse criado por Elkington (1997).

Segundo Gmelin e Seuring (2014), o triple-botton-line é a definição de sustentabilidade ou de interpretação de desenvolvimento sustentável mais aceita, atualmente, e deve satisfazer as seguintes condições: a rentabili- dade econômica, o respeito ao meio ambiente e a responsabilidade social. Na mesma direção, as inovações que buscam atingir esses três objetivos são denominadas inovações sustentáveis. Os autores relacionados utilizaram as qualificações eco, environmental e green e trouxeram os três objetivos da sustentabilidade em suas conceituações, embora, tenham apresentado algumas diferenciações de foco e ênfase em alguma das perspectivas: i) econômico-ambiental (FUSSLER; JAMES,1996); ii) ambiental e sustentável (RENNINGS, 2000); iii) ambiental (KEMP; PEARSON, 2007; OECD, 2009a; OLTRA; SAINTJEAN, 2009; DRIESSEN; HILLEBRAND, 2002; CHEN LAI; WER, 2006); iv) social-ambiental (REID; MIEDZINSKI, 2008; KLEMMER; LEHR; LÖBBE, 1999); v) econômico-ambiental (ANDERSEN, 2008; ARUNDEL; KEMP, 2009; SEVERO et al., 2015).

Rennings (2000) observou que a OCDE, no Manual de Oslo, não distinguiu inovações ambientais e não ambientais e que essa distinção, também, não esteve presente nos estudos empíricos de inovação até então. Assim, a inovação sustentável é motivada pela preocupação com a redução dos impactos ambientais. Posteriormente, a lacuna conceitual foi preenchida pela própria OCDE (2009a; 2009b) quando, em um novo documento, definiu que o conceito de ecoinovação deve ser compreendido e analisado em três dimensões: seus objetivos (foco principal), seus mecanismos (métodos para introdução de mudanças relacionadas aos objetivos) e seus impactos (os efeitos sobre as condições ambientais).

A dimensão objetivos ou metas (targets) são os focos da inovação que podem ter como alvo: i) produtos, que tanto podem ser mercadorias ou serviços; ii) processos, relacionados a procedimentos e métodos de produção; iii) métodos de marketing que tratam da promoção e precificação de produtos e estratégias orientadas para o mercado; iv) organizacionais, relacionadas à estrutura da gestão e distribuição de responsabilidades; v) institucionais, que incluem os organismos sociais, arranjos institucionais, normas sociais e valores culturais. Ou seja, as ecoinovações em produtos e processos estão relacionadas com mudanças tecnológicas, enquanto as demais relacionam-se a mudanças não tecnológicas (OECD, 2009b). 
Os mecanismos estão relacionados aos métodos, segundo os quais as mudanças, que geram ecoinovações, são introduzidas. Quatro são os mecanismos encontrados: i) modificação, que tanto pode ser na produção (pequena ou progressiva), como nos ajustes dos processos; ii) redesign que cuida de mudanças significativas nos produtos existentes, nos processos, nas estruturas organizacionais, dentre outros; iii) alternativas, como a introdução de produtos e serviços que podem cumprir a mesma necessidade funcional e servem como substitutos para outros produtos; iv) criação, concepção e introdução de produtos totalmente novos, processos, procedimentos, organizações e instituições (OECD, 2009a).

Com relação à dimensão impactos, esses demonstram o efeito da ecoinovação no ambiente, através de seu ciclo de vida ou alguma outra área de foco. Os potenciais impactos ambientais resultam do que foi definido nas dimensões anteriores (objetivos e mecanismos) e da sua interação com o ambiente sociotécnico. Dado um alvo específico, a magnitude potencial do benefício ambiental tende a depender do mecanismo da ecoinovação (OECD, 2009a).

\subsection{Direcionadores das ecoinovações}

Nesta parte do estudo, apresentaram-se, primeiramente, as teorias sobre os determinantes das ecoinovações identificados na literatura. Em linhas gerais, as pequenas variações identificadas, entre os autores, decorrem muito mais da forma como cada um deles classifica os fatores determinantes, inexistindo divergências conceituais.

Rennings (2000), ao analisar as características particulares das ecoinovações, estabeleceu três grupos de fatores determinantes, sendo eles:

a) fatores puxados pelo mercado (market pull), como a participação de mercado (market share), a concorrência (competition), a busca de novos mercados (new markets), os custos de mão-de-obra (labor costs), a imagem da organização (image) e a demanda dos consumidores (customer demand);

b) fatores empurrados pela tecnologia (technology push), como a qualidade do produto (product quality), a eficiência dos materiais (material efficiency), a movimentação do produto (product palette) e a eficiência energética (energy efficiency);

c) fatores empurrados pela regulamentação (regulatory push), sendo a legislação ambiental vigente (existing environmental law), os padrões de segurança ocupacional e saúde (occupational safety and health standards) e a futura regulamentação (expected regulation).

Apesar das inovações serem desejadas socialmente, as imperfeições no mercado podem gerar obstáculos ao desenvolvimento pelos agentes privados. Dessa forma, quando os determinantes empurrados pela tecnologia (technology push) e os puxados pelo mercado (market pull) não são, suficientemente, fortes, as ecoinovações necessitam de reforço regulatório para promovê-las (RENNINGS, 1998).

Bernauer et al. (2006) propuseram um quadro conceitual para estudar os determinantes das inovações ambientais, segmentando-os em três grupos: i) os determinantes regulatórios (regulatory determinants), enfatizando questões relacionadas com o rigor (stringency) da regulamentação ambiental atual e a previsibilidade (predictability) de mudanças futuras; ii) os determinantes de mercado (market determinants), com ênfase na competitividade (competitiveness) e benefícios ao consumidor (consumer benefit); iii) fatores internos da firma (firm internal factors), tendo como relevantes as capacidades "verdes" (green capabilities), a inovatividade (inovativeness) e o tamanho da firma (firm size).

Horbach (2008) propôs uma nova classificação, com base em dados coletados na Alemanha, distinguindo: i) fatores do lado da oferta (supply side), como capacidades tecnológicas, incluindo capital humano e conhecimento, e problemas de apropriação dos resultados das inovações (restringir imitações), com base nas estruturas de mercados restritivos (monopólio, por exemplo), tamanho das firmas e ganhos de escala; ii) fatores do lado da demanda (demand side), como as expectativas de demanda de mercado, conscientização ambiental e preferência por produtos, amigavelmente, sustentáveis pela sociedade; iii) fatores políticos e institucionais (institutional and political influences), como política ambiental de incentivo à inovação baseada em incentivos ou abordagens regulatórias e estrutura institucional, ou seja, no 
tocante a oportunidades políticas de grupos ambientalmente orientados, organização de fluxos de informação e existência de redes de inovação.

Kesidou e Demirel (2012), com base em dados coletados junto a empresas britânicas, constataram que fatores da demanda impactam nas decisões das firmas em investir em ecoinovações, alinhando as práticas de negócio com as expectativas sociais e exigências dos consumidores, capacidades organizacionais relacionadas à existência de um sistema de gestão ambiental e o rigor das regulações ambientais. Com relação a esse último aspecto, os autores identificaram que o rigor da regulação afeta de forma diferente as ecoinovações em empresas menos inovadoras, em comparação com as mais inovadoras.

Nesse aspecto, encontra-se uma possibilidade de futuros estudos para verificar como empresas de diferentes perfis de inovação se comportam diante da hipótese de Porter (2009), no que se refere a como a regulação pode impactar, positivamente, a competitividade das empresas inovadoras. O impacto da regulação sobre as inovações ambientais foi discorrido mais, detalhadamente, no tópico seguinte.

Horbach et al. (2012) trouxeram importantes contribuições ao realizaram pesquisa quantitativa na Alemanha para identificar os determinantes das ecoinovações, por tipo de impacto ambiental, premissa que não foi considerada em estudos anteriores. Consideraram determinantes os seguintes fatores: regulação (regulation), fatores puxados pelo mercado (market pull factors), fatores empurrados pela tecnologia (technology push factors) e fatores específicos da firma (firm specific factors). Para a regulação, destacaram o estudo sobre patentes de Popp (2006) que aponta a regulação nacional como o principal direcionador, nos Estados Unidos, Japão e Alemanha. Para os fatores puxados pelo mercado, incluíram a contribuição de Kammerer (2009), ao considerar a importância dos benefícios ao consumidor e ao reconhecerem que não existirem fortes estímulos a ecoinovação do lado da demanda, além da regulação, para superar o problema da dupla externalidade.

Dentre os determinantes empurrados pela tecnologia, destacam as capacidades tecnológicas da firma (knowledge capital) e os sistemas de gestão ambiental. Os fatores específicos da firma são os mecanismos de transferência de conhecimento e o envolvimento em redes de relacionamento (WAGNER, 2009) e as capacidades "verdes" (HART, 1995; KAMMERER, 2009). Os autores reconhecem que a literatura apresenta uma série complexa de diferentes fatores determinantes das ecoinovações, sejam da oferta, da demanda ou específicos da firma. Dentre estes, acentuam o papel da regulação, a redução de custos e os benefícios ao consumidor. A regulação vigente e esperada tem efeito sobre as organizações nas inovações relacionadas a redução de gases, poluição da água, emissão de ruídos, restrições a substituições perigosas e incrementos na reciclabilidade de produtos. As reduções de custos são importantes para motivar a redução de energia e uso de materiais indicando o preço da energia e dos materiais e a tributação como direcionadores das ecoinovações. Os requisitos dos consumidores, como fonte de inovações ambientais, relacionam-se à melhoria da performance ambiental dos produtos e com processos que aumentem a eficiência dos materiais e reduzam o consumo de energia, desperdícios e uso de substâncias perigosas.

$\mathrm{Na}$ pesquisa sobre os drivers de diferentes tipos de ecoinovação em pequenas e médias empresas europeias, Triguero et al. (2013) apresentaram, detalhadamente, os fatores a serem considerados: i) do lado da oferta, os determinantes dividem-se em empurrados pela tecnologia (capacidades tecnológicas e gerenciais, colaboração com institutos de pesquisa, agencias e universidades, acesso ao conhecimento e informações externas) e pela redução de custo (tamanho da firma, preço dos materiais e preço da energia); ii) do lado da demanda, segmentados em fatores puxados pelo mercado (participação de mercado e demanda de mercado por produtos verdes); iii) fatores puxados/empurrados pela regulação (regulações existentes, futuras regulações esperadas e acesso aos subsídios e incentivos fiscais existentes).

Finalmente, Cuerva et al. (2014) apresentaram o resultado de sua pesquisa quantitativa realizada junto a empresas espanholas de alimentos e bebidas, a fim de testarem se os drivers para inovações "verdes" e "não verdes" são os mesmos. Ao mesmo tempo, 
tomando por base Horbach (2008) e Horbach et al. (2012), apresentaram sua contribuição pessoal em um quadro conceitual sobre os determinantes de ecoinovações em pequenas e médias empresas, dentre as quais se destaca que uma das razões poderia ser a predominância de tecnologias incrementais na maioria das inovações ambientais implementadas, que requerem pouco esforço de pesquisa e desenvolvimento (P\&D).

Além disso, outros resultados da pesquisa de Cuerva et al. (2014) destacaram que: i) o capital humano, também, promove inovações verdes em menor grau que em outras inovações, confirmando o resultado de trabalhos empíricos anteriores; ii) as restrições financeiras limitam as inovações verdes em maior grau que em outras inovações; iii) com relação às capacidades organizacionais, sistemas de gestão da qualidade promovem inovações verdes em maior grau que outras inovações. A pesquisa apresentou coeficientes positivos e significativos para este quesito; iv) as inovações verdes dependem parcialmente das pressões de mercado. Embora a diferenciação de produto seja uma característica significativa de inovação verde, a sua adoção depende também da consciência do cliente que, por questões de conservadorismo, pode não mudar sua preferência.

Cuerva et al. (2014), ainda, ressaltaram que as práticas de responsabilidade ambiental, em pequenas e médias empresas e certificações de origem, não influenciam, positivamente, as inovações "verdes", embora o façam para inovações convencionais. O comportamento das organizações em questões de natureza social, ética e legal melhora a imagem da empresa, mas não necessariamente em questões ambientais, na mesma linha de pensamento de Kesidou e Demirel (2012), podendo impactar positivamente grandes empresas (WAGNER, 2010). A certificação de origem não é relevante para ecoinovação, pois os produtos procedentes de regiões certificadas estão protegidos pela reputação e conceito restringindo a preferência e a concorrência com produtos não autênticos.

Em relação aos recursos públicos na promoção das ecoinovações, estes não são consenso como fator determinante. De Marchi (2012) e Horbach (2008) concluíram que a influência sobre as ecoinovações é positiva, porém refutada, dentre outros, por Kammerer (2009) e Trigueiro et al. (2013). Esses resultados divergentes abriram a oportunidade de uma revisão da teoria para verificar se o modelo da tríplice hélice de Etzkowitz e Leydesdorff (2000), que considera a integração dos esforços do estado, da indústria e da academia na promoção das inovações, é válido para as ecoinovações em qualquer circunstância ou, somente, em condições específicas.

A cooperação entre concorrentes, fornecedores e clientes, centros de pesquisas e universidades não são significantes para qualquer tipo de inovação, segundo Cuerva et al. (2014). A cooperação em pequenas e médias empresas desencoraja a inovação em indústrias que oferecem produtos homogêneos. Os resultados desta pesquisa abrem as portas para um novo estudo sobre a teoria da Open Innovation de Chesbrough (2003), que preconiza a cooperação entre os agentes da inovação, verificando em que circunstâncias e grau se aplicam as inovações ambientais, e o tamanho da firma influencia positivamente ambos os tipos de inovação confirmando a posição de outros autores, dentre eles De Marchi (2012), Bernauer et al (2006), Cleff e Rennings (1999).

\subsection{Papel da regulação das ecoinovações}

Na sequência, discorreu-se sobre alguns aspectos teóricos particulares relacionados à regulação enquanto direcionador de inovações ambientais, por se tratar de um aspecto da mais alta relevância. Especificamente sobre as questões regulatórias, a legislação é um importante direcionador que distingue as inovações ambientais das não ambientais, conforme constatado nos referenciais teóricos pesquisados (RENNINGS, 2000; BERNAUER et al., 2006; HORBACH, 2008; KESIDOU; DEMIREL, 2012; HORBACH et al., 2012; TRIGUERO et al., 2013; CUERVA et al., 2014).

A partir das proposições de Brundtland (1987), a regulação sobre questões ambientais passa a impactar o dia a a dia das organizações como uma forma de promover mudanças para esse novo paradigma. Porter e Van der Linde (1995a) afirmam que a necessidade de uma regulamentação para proteger o meio ambiente tem uma aceitação generalizada, embora de má vontade, devido à crença de 
que as regulamentações ambientais corroem a competitividade. Os autores defendem uma nova abordagem, quebrando o paradigma do conflito ecologia versus economia, no qual o foco da inovação deve estar nos resultados e não na tecnologia e, assim, inovar para atender às normas pode trazer compensações ao se usar melhores insumos, criando-se produtos melhores ou melhorando a rentabilidade dos produtos. A regulação ambiental, segundo a visão dos autores, atende a seis propósitos: i) mostrar as empresas a provável ineficiência de recursos e o potencial das melhorias tecnológicas; ii) trazer mais benefícios através da coleta de informações, incrementando a consciência corporativa; iii) reduzir a incerteza para a realização de investimentos; iv) pressionar a empresa para que realize inovações; v) nivelar o desempenho operacional das empresas, promovendo uma proteção ao evitar ganhos oportunistas de empresas que evitam investimentos ambientais; vi) melhorar a qualidade ambiental enquanto as inovações não compensarem os custos de conformidade (PORTER; VAN DER LINDE, 1995b).

Corroborando com o mesmo pensamento, Nidumolu et al. (2009) afirmam que a sustentabilidade não corrói os lucros como se acredita, mas, pelo contrário, pode reduzir custos e incrementar vendas, pois, segundo os autores, a sustentabilidade deveria ser a referência para toda inovação, e somente as organizações que tiverem a sustentabilidade como objetivo alcançarão vantagem competitiva. Isto porque a sustentabilidade forçará as empresas a mudarem sua maneira de pensar sobre produtos, tecnologias, processos e modelos de negócios. Rennings (2000), ao propor a sua visão sobre os determinantes das ecoinovações, afirma que, quando fatores empurrados pela tecnologia (technology push) e fatores puxados pelo mercado (market pull) não são suficientemente fortes, existe a necessidade de suporte regulatório específico. Nidumolu et al. (2009) também consideram a adequação e o respeito às normas ambientais o primeiro de cinco estágios em que as empresas devem seguir ao buscarem a sustentabilidade como a base da inovação.

Por ser um tema complexo pela diversidade de legislações existentes entre países e nos mais diversos níveis, Rennings (2000) reconhece que, para que a regulação favoreça o surgimento de ecoinovações, é necessário que a política ambiental e as políticas de incentivos às inovações ambientais estejam alinhadas. Isto significa dizer que se a política ambiental é restritiva; ao inibir os danos ambientais, deve existir uma política de incentivo às ecoinovações que busque alternativas que promovam melhorias para o meio ambiente e para a sustentabilidade.

\section{METODOLOGIA}

Tratou-se de uma pesquisa qualitativa com utilização de abordagem documental dos referenciais teóricos abordados destacando as principais características e os fatores direcionadores e determinantes das ecoinovações que levam ao desenvolvimento sustentável nas organizações.

A técnica escolhida, para a análise das informações, foi a bibliometria. Assim, segundo Ginexi e Hilton (2006), procurou-se assegurar os princípios de uma pesquisa bibliométrica, identificando a qualidade da contribuição científica por meio de artigos a partir do impacto na ciência em longo prazo, bem como a avaliação das revistas por meio de índices de impacto, por meio da indexação em bases de dados, de modo que permitisse a aquisição de um corpo teórico coeso na fundamentação em relação ao objeto de análise a que se propõe esta pesquisa. O levantamento do referencial teórico foi realizado no metabuscador Scopus. As áreas de conhecimento pesquisadas nas bases de dados foram Business, Management and Accounting. Numa primeira rodada, foi feita uma pesquisa avançada, utilizando-se as palavras-chave green innovation, eco-innovation, environmental innovation e sustainable innovation, a fim de conceituar e caracterizar a presença desta diversidade de conceitos na literatura, no período de julho de 2014 a março de 2015.

Constatou-se que todos são temas de estudo e pesquisa multidisciplinares sendo estudados em publicações relacionados à Gestão e Negócios, Economia Ecológica e Engenharia. Tal evidencia justifica a existência de múltiplos termos para expressar conceitos sinônimos e intercambiáveis. Em cada uma das buscas foram analisados os resumos com a finalidade de verificar se o conteúdo dos 
artigos guardava relação e aderência com o objetivo do presente estudo, ou seja, de compor uma abordarem dos determinantes e direcionadores das inovações ambientais. Assim a partir dos elementos foi composto um quadro conceitual promovendo um direcionamento teórico de possibilidades de análise. A confiabilidade do referencial teórico deste trabalho está fundamentada nos índices métricos das revistas pesquisadas, conforme Quadro 1.

Quadro 1 - Periódicos pesquisados que serviram de base para a pesquisa

\begin{tabular}{|l|c|c|c|}
\hline \multirow{2}{*}{\multicolumn{1}{|c|}{ Periódicos }} & \multicolumn{2}{|c|}{ JCR } & SCIMAGO \\
\cline { 2 - 4 } & Fator de Impacto & $\begin{array}{c}\text { Fator de Impacto - } \\
\mathbf{5} \text { anos }\end{array}$ & Índice H \\
\hline Ecological Economics & 2.517 & 4.002 & 103 \\
\hline Energy Policy & 2.696 & 3.402 & 92 \\
\hline Environmental Policy and Governance & 1.117 & - & 25 \\
\hline Harvard Business Review & 1.831 & 2.070 & 104 \\
\hline Journal of cleaner production & 3590 & 4.088 & 69 \\
\hline Journal of Business ethics & 1.552 & 1.889 & 75 \\
\hline Journal of environmental economics and management & 2.522 & 3.398 & 75 \\
\hline R \& D management & 1.266 & 2.635 & 56 \\
\hline Research Policy & 2.598 & 3.989 & 129 \\
\hline Technological Forecasting and Social Change & 1.959 & 2.405 & 52 \\
\hline The Academy of management review & 7.817 & 9.698 & 163 \\
\hline
\end{tabular}

Fonte: Extraído do Journal Citations Report (JCR) e SCImago Journal \& Country Rank

Da mesma forma, foram considerados dados relevantes de fontes como revistas acadêmicas, livros, dissertação, relatórios de fontes do governo e de organizações internacionais e internet. Os dados das fontes consultadas foram intencionalmente selecionados com base em sua relevância para o tema em discussão. O estudo adotou a retrospectiva da abordagem da literatura e análise de conteúdo dos materiais relevantes compondo a trajetória de elementos e fatores preponderantes para os temas desta pesquisa.

\section{RESULTADOS E DISCUSSÃO}

Após análise dos referenciais teóricos, concluiu-se que os determinantes das ecoinovações apresentaram pequenas variações, de um autor para outro, e decorrentes dos objetivos e focos específicos, ou mesmo especificidades dos setores ou dos países, nos quais as pesquisas foram aplicadas, sendo pertinente a elaboração de um quadro conceitual que consolidasse todos os direcionadores, em consonância com as visões dos diferentes autores.
Para a elaboração do esquema conceitual, os fatores determinantes foram elaborados em uma nova categorização, de acordo com a sua natureza, com vistas a unificar a terminologia adotada pelos autores pesquisados, cuja proposição está apresentada na Quadro 2 , contemplando os seguintes fatores determinantes: mercadológicos, tecnológicos e de produção, organizacionais e de gestão, econômicos, políticos e institucionais e regulatórios. 
Quadro 2 - Fatores determinantes das eco-inovações baseados na bibliografia

\begin{tabular}{|c|c|c|}
\hline $\begin{array}{c}\text { Categorização } \\
\text { dos fatores } \\
\text { determinantes }\end{array}$ & Discriminação & Autor \\
\hline Mercadológicos & $\begin{array}{l}\text { Participação de mercado (Market share) } \\
\text { Concorrência } \\
\text { Competitividade } \\
\text { Imagem da empresa } \\
\text { Demanda por produtos verdes/pressão de mercado } \\
\text { Busca de novos mercados } \\
\text { Benefícios ao consumidor } \\
\text { Expectativas do Mercado }\end{array}$ & $\begin{array}{l}\text { Rennings, 2000; Triguero } \\
\text { et al., 2013; Bernauer et } \\
\text { al., 2006; } \\
\text { Horbach,2008; Kesidou e } \\
\text { Demirel, 2012; Triguero et } \\
\text { al., 2013; } \\
\text { Horbach et al., } 2012\end{array}$ \\
\hline $\begin{array}{c}\text { Tecnológicos e de } \\
\text { produção }\end{array}$ & $\begin{array}{l}\text { Qualidade do produto } \\
\text { Eficiência dos materiais } \\
\text { Movimentação do produto } \\
\text { Eficiência energética } \\
\text { Capacidades tecnológicas (inclui capital humano e } \\
\text { conhecimento) } \\
\text { Pesquisa e desenvolvimento } \\
\text { Cooperação (concorrentes, fornecedores, clientes) } \\
\text { Mecanismos de transferência do conhecimento } \\
\text { Redes de relacionamento (institutos de pesquisa, } \\
\text { universidades) } \\
\text { Acesso ao conhecimento e informações externas }\end{array}$ & $\begin{array}{l}\text { Rennings, 2000; } \\
\text { Horbach, 2008; Horbach } \\
\text { et al., 2012; Triguero et al., } \\
\text { 2013; Cuerva et al., } 2014\end{array}$ \\
\hline $\begin{array}{c}\text { Organizacionais e } \\
\text { de gestão }\end{array}$ & $\begin{array}{l}\text { Capacidades "verdes" } \\
\text { Inovatividade } \\
\text { Responsabilidade Social corporativa } \\
\text { Sistema de gestão ambiental } \\
\text { Capacidades gerenciais }\end{array}$ & $\begin{array}{l}\text { Bernauer et al., 2006; Hor- } \\
\text { bach et al., 2012; Kesidou } \\
\text { e Demirel, 2012; Cuerva } \\
\text { et al., 2014; } \\
\text { Triguero et al., } 2013\end{array}$ \\
\hline Econômicos & $\begin{array}{l}\text { Custos da mão-de-obra } \\
\text { Tamanho da firma } \\
\text { Ganhos de escala } \\
\text { Preço dos insumos (materiais e energia) } \\
\text { Restrições Financeiras } \\
\text { Conscientização ambiental } \\
\text { Preferencias por produtos sustentáveis }\end{array}$ & $\begin{array}{l}\text { Rennings, 2000; } \\
\text { Bernauer et al., 2006; Hor- } \\
\text { bach, 2008; Triguero et al., } \\
\text { 2013; Cuerva et al., } 2014\end{array}$ \\
\hline $\begin{array}{c}\text { Políticos e } \\
\text { Institucionais }\end{array}$ & $\begin{array}{l}\text { Políticas de incentivo e subsídios } \\
\text { Estrutura institucional } \\
\text { Redes de inovação }\end{array}$ & $\begin{array}{l}\text { Horbach, 2008; Triguero } \\
\text { et al., 2013; Cuerva et al., } \\
2014\end{array}$ \\
\hline Regulatórios & $\begin{array}{l}\text { Legislação ambiental vigente } \\
\text { Padrões de segurança e saúde ocupacional } \\
\text { Rigor da legislação vigente } \\
\text { Expectativas e previsibilidade da legislação futura } \\
\text { Apropriação e proteção às inovações (marcas e patentes) }\end{array}$ & $\begin{array}{l}\text { Rennings, 2000; Triguero } \\
\text { et al., 2013; Bernauer } \\
\text { et al., 2006; Kesidou e } \\
\text { De-mirel, 2012; Horbach, } \\
\text { 2008; Horbach et al., } 2012\end{array}$ \\
\hline
\end{tabular}

Fonte: elaboração própria.

Nos resultados encontrados, o trabalho de Cuerva et a.1 (2014) foi o que trouxe aspectos significativos contrapondo posições teóricas, amplamente, aceitas entre os pesquisadores de inovação. Especificamente, os resultados demonstram que não validam amplamente a posição de Etzkowitz e Leydesdorff (2000) sobre a tríplice hélice nem a de Chesbrough (2003) sobre Inovação Aberta, quando aplicadas às inovações sustentáveis.
Quanto a tríplice hélice, os autores encontraram resultados divergentes em pesquisas anteriores sobre o uso de recursos públicos na promoção de ecoinovações, o que contraria a teoria. Outro aspecto, a ser verificado no contexto da inovação aberta, referiu-se à cooperação entre concorrentes, clientes, fornecedores institutos de pesquisa e universidades como um fator de relevante importância para a promoção de inovações. 
O resultado da pesquisa, porém, identificou a cooperação como um fator não significante, o que diverge da teoria. Sobre esses dois aspectos, recomenda-se uma análise mais aprofundada, em razão de não se aplicarem, plenamente, às ecoinovações.

Neste estudo, constatou-se que ecoinovações e desenvolvimento sustentável são temas intimamente relacionados, de forma que o desenvolvimento sustentável é o marco referencial que propugna a inovação como mecanismo de desenvolvimento de novos produtos onde questões ambientais como a redução do consumo de energia, a utilização racional dos recursos naturais, a substituição de matérias primas, degradação do meio ambiente, destinação de resíduos e poluição são desejados pelo mercado gerando mudanças crescentes nos gostos e preferencias dos consumidores, ensejando novas abordagens de marketing na busca de participações de mercado e de vantagem competitiva realimentando a competição e as inovações e considerando novas variáveis para a avaliação da performance do mercado e do produto. Essas mudanças exigem da empresa adequação às normas e regulamentações ambientais que devem submeter e adequar seus produtos.

De forma geral, os fatores determinantes e direcionadores das inovações ambientais apresentaram similaridades entre os autores, havendo referência explícita nos trabalhos de Rennings $(1998 ; 2000)$ sobre a importância dos marcos regulatórios, quando imperfeições de mercado não são suficientes para promover o desenvolvimento das inovações. O sucesso de sua adoção é devido ao alinhamento entre as medidas de política ambiental e a política de inovações. Diferentes autores (RENNINGS 1998; 2000; BERNAUER et al., 2006; HORBACH, 2008; HORBACH et al., 2012; KESIDOU; DEMIREL, 2012; TRIGUERO et al., 2013) fazem referência a hipótese de Porter (PORTER; VAN DER LINDE, 1995a; 1995b), que preconiza a regulação como fator de incentivo à competitividade das empresas. Também aqui a literatura pesquisada apresenta afirmativas empíricas (através da observação) sobre a competitividade das firmas, a partir das quais podem se originar estudos futuros, de natureza qualitativa ou quantitativa.

Outra importante característica observada na literatura pesquisada, é que a maioria dos autores também apontam os direcionadores de forma empírica, cabendo a Horbach et al. (2012) a originalidade de uma pesquisa sobre os determinantes das ecoinovações por tipo de impacto ambiental, tema este que poderia ser aprofundado em pesquisas futuras.

As ecoinovações apresentam algumas particularidades em relação às inovações convencionais que proporcionam novas oportunidades de estudos e pesquisas para verificar a aplicabilidade de referenciais teóricos já consagrados para as inovações em geral no contexto das inovações ambientais.

A pesquisa de Cuerva et al. (2014) apresentou dois resultados bastante significativos. O primeiro deles é o resultado com outras pesquisas de serem ou não os recursos públicos um fator determinante das ecoinovações. Os autores apresentam resultados divergentes com outras pesquisas, o que pode ensejar novos estudos no sentido de se verificar em que contexto ou situações específicas a tríplice hélice de Etzkowitz e Leydesdorff (2000) é explicada para as ecoinovações. O segundo refere-se à baixa cooperação entre stakeholders para o desenvolvimento de ecoinovações. Neste contexto, estudos futuros podem trazer novas perspectivas sobre a aplicabilidade da teoria da inovação aberta de Chesbrough (2003) nas ecoinovações.

\section{CONSIDERAÇÕES FINAIS}

$\mathrm{O}$ artigo procurou identificar as principais características e os fatores determinantes das ecoinovações identificados na literatura. Os achados foram consolidados e agrupados em categorias, a saber: mercadológicos, econômicos, políticos e institucionais, regulatórios, organizacionais e de gestão tecnológicos e de produção.

Com base no material analisado, sugeriram-se as seguintes proposições: i) a partir dos fatores determinantes elencados, identificar quais deles são mais impactantes no desenvolvimento de ecoinovações para a obtenção de vantagens competitivas nas organizações; ii) pesquisar contextos e fatores específicos em que o modelo da tríplice hélice de Etzkowitz e Leydesdorff (2000) não se aplicam, plenamente, às ecoinovações; iii) pesquisar a aplicabilidade e possíveis fatores limitantes da teoria da inovação aberta, de Chesbrough (2003) quando aplicada às ecoinovações. 
Dentre as limitações deste estudo, a falta de identificação dos condicionantes ao desenvolvimento de inovações ambientais representou uma possibilidade de estudos futuros, uma vez que não foram identificadas nas bases de dados pesquisadas estudo que viabilizasse essas relações. Além disso, outra limitação foi a ausência de uma etapa quantitativa que permitisse mensurar de forma mais ampla as relações entre os temas elencados, constituindo, assim, uma oportunidade de trabalho futuro, onde as atribuições de pesos às categorias elencadas ensejariam o conhecimento, acerca de quais elementos obtiveram maior relevância, elaborando um método de equações estruturais, consolidando os fatores às categorias que impulsionam as ecoinovações, no contexto empírico.

\section{REFERÊNCIAS}

ANDERSEN, M. M. Eco-innovation-towards a taxonomy and a theory. In: CONFERENCE 2008 ON ENTREPRENEURSHIP AND INNOVATION ORGANIZATIONS, INSTITUTIONS, SYSTEMS AND REGIONS. 25TH CELEBRATION DRUID, 17-20 jun. 2008, Copenhage, Dinamarca. Anais... Copenhage: CBS, 2008.

ARUNDEL, A.; KEMP, R. Measuring eco-innovation. UNU-MERITWorking Paper Series, Maastricht, n. 17, 2009.

BAUMANN, H.; BOONS, F.; BRAGD, A. Mapping the Green Product Development Field: Engineering, Policy and Business Perspectives. Journal of Cleaner Production, Gotemburgo, v. 10, n. 5, p. 409-425, 2002.

BERNAUER, T.; ENGELS, S.; KAMMERER, D.; SEIJAS, J. Explaining Green Innovation: Ten Years after Porter's Win-Win Proposition: How to Study the Effects of Regulation on Corporate Environmental Innovation? CIS Working Paper, Zurich, n. 17, 2006.

BOONS, F.; MONTALVO, C.; QUIST, J.; WAGNER, M. Sustainable innovation, business models and economic performance: an overview. Journal of Cleaner Production, Gotemburgo, v. 45, p. 1-8, abr. 2013.

BOONS, F.; LÜDEKE-FREUND, F. Business models for sustainable innovation: state-of-the-art and steps towards a research agenda. Journal of Cleaner Production, Gotemburgo, v. 45, p. 9-19, 2013.

BRUNDTLAND, G. H. Report of the World Commissionon Environment and Development: Our Common Future. World Commission on Environment and Development. New York: United Nations, mar. 1987.

CHEN, Y. S., LAI, S. B., AND WEN, C. T. The influence of green innovation performance on corporate advantage in Taiwan. Journal of Business Ethics, v. 67, n. 4, p. 331-339, 2006.

CHESBROUGH. H. W. The era of open innovation. MIT Sloan Management Review, v. 44, p. 35-41, 2003.

CLEFF, T., RENNINGS, K. Determinants of environmental product and process innovation. European
Environment (special issue) in Environmental Policy and Governance, v. 9, n. 5, p. 191-201, 1999.

CUERVA, M. C., TRIGUERO-CANO, A., CÓRCOLES, D. Drivers of green and non-green innovation: empirical evidence in Low-Tec SMEs. Journal of Cleaner Production, Gotemburgo, v. 68, p. 104-113, 2014.

DE MARCHI, V. Environmental innovation and R\&D cooperation: empirical evidence from Spanish manufacturing firms. Research Politics, v. 41, p. 614-623, 2012.

DRIESSEN, P.; HILLEBRAND, B. Adoption and diffusion of green innovations. In: Nelissen, W. and Bartels, G. (Ed.). Marketing for sustainability: towards transactional policy-making. Amsterdam: IOS Press Inc., 2002. p. 343-355.

ELKINGTON, J. Cannibals with forks: the triple bottom line of the 21st century business. Stoney Creek: New Society Publishers, 1997.

ETZKOWITZ, H.; LEYDESDORFF, L. The dynamics of innovation: from National Systems and "Mode 2" to a Triple Helix of university-industry-government relations. Research Policy, v. 29, n. 2, p. 109-123, fev. 2000.

FADHILAH, Z.; RAMAYAH, T. Behind the Green Doors: what management Practices Lead to Sustainable Innovation? Procedia Social and Behavioral Sciences, v. 65, p. 247-252, dez. 2012.

FUSSLER, C.; JAMES, P. Driving Eco-Innovation: a breakthrough discipline for innovation and sustainability. London: Pitman, 1996.

GMELIN, H.; SEURING, S. Determinants of a sustainable new product development. Journal of Cleaner production, v. 69, p. 1-9, 2014.

GINEXI, E. M., HILTON, T. F. What's next for translation research? Evaluation Health Professional, v. 29, n. 3, p. 334-347, 2006.

HART, S. L. A natural resurce-based view of the firm. Academy of Management Review, v. 20, n. 4, p. 986-1014, 1995.

HORBACH, J. Determinants of environmental innovation - new evidence from German panel data sources. Research Policy, v. 37, p. 163-173, 2008.

HORBACH, J.; RAMMER, C.; RENNINGS, K. Determinants of eco-innovation by type of environmental impact - the role of regulatory push/pull, technology push and Market pull. Ecological Economics, v. 78, p. 112-122, 2012.

HUANG, Y. C.; WU, Y. C. J. The Effects of organizational factors on Green New Product Success: evidence from high-tech industries in Taiwan. Managing Decision, v. 48, n. 10, p. 1539-1567, 2010.

KAMMERER, D. The efects of customer benefit and regulation on environmental product innovation. Empirical evidence from appliance manufacturers in Germany. Ecological Economics, v. 68, p. 2285-2295, 2009.

KEMP, R.; PEARSON, P. Final report of the MEI project measuring eco innovation. One Merit Maastricht, 2007. Disponível em: <http://www.merit.unu.edu/MEI/ deliverables / MEI\% 20D15\% 20Final \% 20report \% 20 about $\% 20$ measuring\%20eco-innovation.pdf>. Acesso em: 12 jan. 2015. 
KESIDOU, E.; DEMIREL, P. On the drivers of ecoinnovations: empirical evidence from the UK. Research Policy, v. 41, p. 862-870, 2012.

KLEMMER, P.; LEHR, U.; LÖBBE, K. Environmental Innovation: incentives and barriers (Innovation Effects of Environmental Policy Instrument). Berlin, Germany: Analytica Verlag, 1999. p. 139.

MEDEIROS, J. F.; RIBEIRO, J. L. D.; CRUZ, C. M. L. Inovação ambientalmente sustentável e fatores de sucesso na percepção de gestores da indústria de transformação. Cadernos EBAPE.BR, v. 10, n. 3, p. 652-676, 2012.

NIDUMOLU, R.; PRAHALAD, C. K.; RANGASWAMI, M. R. Why sustainability is now the key driver of innovation. Harvard Business Review, set. 2009.

ORGANIZAÇÃOPARACOOPERAÇÃOECONÔMICA E DESENVOLVIMENTO - OCDE. Manual de Oslo - diretrizes para coleta e interpretação de dados sobre inovação. 3. ed. FINEP, 1997.

OLTRA, V.; SAINT JEAN, M. Sectoral systems of environmental innovation: an application to the French automotive industry. Technological Forecasting and Social Change, v. 76, p. 567-583, 2009.

ORGANISATION FOR ECONOMIC CO-OPERATION AND DEVELOPMENT - OECD. The Future of EcoInnovations: the role of business models in green Transformation. Paris: OECD, 2012.

Sustainable Manufactirung and Eco-innovation: framework, practices and measurement - Synthesis Report. Paris: OECD, 2009a.

Eco-innovation in industry: enabling green growth. Paris: OECD, 2009b.

POPP, D. International Innovation and difusuion of air pollution control technologies: the effects of $\mathrm{NO}_{\mathrm{x}}$ and $\mathrm{SO}_{2}$ regulation in the US, Japan, and Germany. Journal of Environmental Economics and Management, v. 51, n. 1, p. 46-71, 2006.

PORTER, M. Competição. 2. ed. rev. e ampl. Rio de Janeiro: Elsevier, 2009. 546p.

PORTER, M.; VAN DER LINDE, C. Green and competitive: ending the stalemate. Harvard Business Review, set./out. 1995a.

. Toward a new conception of environment-competitiveness relationship. Journal of Economic Perspectives, v. 9, n. 4, p. $97-118,1995$ b.
REID, A.; MIEDZINSKI, M. Eco-innovation - final report for Sectoral Innovation Watch. Final report to Europe INNOVA iniative. Technopolis Group, 2008. Disponível em: <http:/ / www.technopolis-group.com/ cms.cgi/site/downloads/index.htm>. Acesso em: 25 mar. 2015.

RENNINGS, K. Redefining innovation - eco-innovation research and the contribution from ecological economics. Ecological Economics, v. 32, p. 319-332, 2000.

. Towards a theory and policy of eco-innovationneoclassical and (co)evolucionary perspectives. ZEW Discussion Papers, Mannheim, n. 98-24, 1998.

SCHIEDERING, T.; TIETZE, F.; HERSTATT, C. Green innovation in technology and innovation management - an exploratory literature review. $R \mathcal{E} D$ Management, v. 42, n. 2, 2012.

SCHUMPETER, J. A. Capitalismo, socialismo e democracia. Rio de Janeiro: Fundo de Cultura, 1961.

SEMAN, N. A. A.; ZAKUAN, N.; JUSOH, A.; ARIF, M. S. M.; SAMAN, M. Z. M. The relationship of green supply chain management and green innovation concept. Procedia-Social and Behavioral Sciences, n. 57, p. 453-457, 2012.

SEVERO, E. A.; GUIMARÃES, J. C. F.; DORION, E. C. $\mathrm{H}$; NODARI, C. H. Cleaner production, environmental sustainability and organizational performance: an empirical study in the Brazilian Metal-Mechanic industry. Journal of Cleaner Production, v. 96, p. 118125, 2015.

TRIGUERO, A.; MORENO-MONDEJAR, L.; DAVIA, M. A. Drivers of diferente types of eco-innovation in European SMEs. Ecological Economics, v. 92, p. 25-33, 2013.

WAGNER, M. The role of corporate sustainability performance for economic performance: a firm-level analysis of moderation effects. Ecological Economics. v. 69, n. 7, p. 1553-1560, maio 2010.

. Erfolgsfaktoren für Nachhaltigkeitsinnovationen: qualitative und quantitative Befunde. Zeitschrift für Umweltpolitik und Umweltrecht - ZfU, v. 2, p. 179-198, 2009.

WORLD BUSINESS COUNCIL FOR SUSTAINABLE DEVELOPMENT - WBCSD. Eco-Efficiency: creating more value with less impact. Geneva: WBCSD, 2000.

Obs.: O autor Pedro Gilberto Aloise é Bolsista do Programa RH Doutorado da Fundação de Amparo à Pesquisa do Amazonas (FAPEAM), Amazonas, Brasil. 